\title{
Biological Control of Insect Pests in Vegetable Crops: An Eco-friendly Approach
}

\author{
P. Pati ${ }^{1 *}$, S.K. Behera ${ }^{2}$, S. Raghu ${ }^{2}$ and M. Annamalai ${ }^{2}$ \\ ${ }^{1}$ Department of Agricultural Entomology, Institute of Agricultural Sciences (IAS), \\ Siksha 'O' Anusandhan (SOA) Deemed to be University, Bhubaneswar, Odisha, India \\ ${ }^{2}$ Crop Protection Division, ICAR-National Rice Research Institute (NRRI), Cuttack-753006, \\ Odisha, India \\ *Corresponding author
}

\section{A B S T R A C T}

\section{Keywords}

Insect pests,

Vegetable crops, Biological control,

Eco-friendly

management

Article Info

\section{Accepted:}

12 December 2020

Available Online:

10 January 2021
Insect pests are the most destructive constraints to world agriculture. They damage millions of hectares of crops belonging to food grains, horticulture crops every year. The extent of damage is increasing year after year under the influence of changing climatic conditions. The developing economies suffer more due to outbreak of insect pests in fruits and vegetables which are the major source of international trade. India produces almost all the vegetables which are consumed inside and exported to other countries. But, insect pests create a major barrier to production and productivity as they are causing several outbreaks. Chemical pesticides are being used to manage these insect pests in order to achieve quick control. But, in many cases, misuse of pesticides led to environmental pollution along with population resurgence, pesticidal residue problem in soil and water, and pest resistance to these chemical pesticides. In such situation, biological control gives a promising hope towards sustainable pest management. Biological control is the, usage of living organisms and their products alternative to chemical pesticides or integrating as one of the component in integrated pest management program. Here, we can employ various biological control agents like natural enemies (predators and parasitoids), entomopathogenic fungi, bacteria, virus, nematodes, protozoa and actinomycetes or their products for effective pest management. They can be directly used or formulated into a commercial formulation suitable for various methods of application. Classical biological control is well-tried, cost effective approach to manage various pests in vegetable crops. Since we use native strains of biocontrol agents, they will be cost effective, more effective due to acclimatization to introduced microclimate and keeps the pest level below acceptable economic threshold levels. In order to reduce the serious problems of chemical pesticides on environment and human life biocontrol is suitable approach. Vegetables being high value, low volume crops and shares major portion of our daily diet needs to be grown pest free and residue free. In order to achieve sustainable pest control and reduce cost of protection, biological control is well suited for management of insect pests in vegetable crops. 


\section{Introduction}

India is the major agriculture based country where more than $60 \%$ of the population live in rural areas and engaged in agriculture. Indian agriculture sector contributes tremendously towards national GDP their by nation's economy. India produces almost all the crops starting from food grains, horticulture crops and commercial crops (Vanitha et al., 2013; APEDA 2020). Number of horticultural products including fruits, vegetables and flowers being exported to other countries earning significant foreign exchange. Among horticulture crops, vegetables contribute to a significant proportion of area and production with major crops like brinjal, tomato, okra, cabbage, potato, onion and cucurbits are being cultivated in the country throughout the crop seasons (NHB 2018). Vegetables contain vital source of proteins, vitamins, minerals, dietary fibers, micronutrients, antioxidants and phytochemicals in our daily diet. They provide nutrition our very diet apart from this they contain array of potential phytochemicals like anti-carcinogenic principles and antioxidants like flavonaoides, glucosinolates and isothyocyanates which will help in combating various disorders. Though production and productivity of vegetable crops increased, the cultivation of these crops facing a number of constraints like pest, diseases and other abiotic factors. Among them, insect pests attack vegetable crops at various crop stages and cause significant reduction in yield and quality (Sharma et al., 2013). They can damage vegetables both in protected structures (net house, polyhouse) and open field with variable damage (Rai et al., 2014). Vegetables like brinjal, cabbage, tomato, potato, chillies, onion and cucurbits infested by majority of insects. Aphids, Jassids, Whiteflies, fruit and shoot borer, hadda beetle, mealybug, leaf minor, tubermoth, head borer and leaf hoppers are major pests causing maximum damage at different crop growth stages.Eevnough, there are a number of methods available to control the damage, usage of chemical pesticides is being followed in a large scale especially during post green revolution years. But the unscientific and indiscriminate use of chemical pesticides brought into various problems like residues in products, harmful effects on human and animals along with environmental pollution. On the other hand, various reports from different researchers that, most of the insect pests developed resistance against major insecticides. Resurgence of the pests also being notices in many parts of the country. This has raised a serious concern among researchers and growers to look into alternative/corrective measures of pest control to achieve sustainable crop protection, production and environmental safety.

One such option is the biological control which eventually attained global preference over synthetic pesticides for effective and eco-friendly management of insect pests in vegetables. Here living organisms and their products are used to maintain pest populations below economic threshold levels (ETL) which also protect natural enemies (Altieri et al., 2005; Mahr et al., 2008).

Significant research and development has taken up during past few decades for biological control of insect pests. Over the past 50 years, biological control remains as one of the component of IPM and showing a steady but promising growth in IPM (Orr 2009). Looking into the importance of biological control, literature survey was carried out and review was made on various pests of major vegetable crops like the extent of damage and their potential management using biological control agents. The detailed literature has been provided in following paragraphs. 
Major insect pests of vegetable crops and their extent of damage in India

Vegetable crops encounter by a number of insect pest all along their growth and development period. They are the major constraints to vegetables both on and off the field. They cause various types of damage based on the feeding behavior. Apart from direct damage, they also act as vectors of number of viral diseases through persistent, semi-persistent and non-persistent manner (Rai et al., 2014). Various researchers estimated crop losses to the tune of $30-40 \%$ at different crop growth stages (Rai et al., 2014). Changes in cultivation practices, like intensive mono cropping, introduction of high yielding but susceptible varieties/hybrids which uses high inputs under changing climatic scenario are the major factors leading to a pest outbreak and epidemics. In some of the situations, minor pests emerged as major problem due to the shift in seasons and favorable environmental conditions (Vanitha et al., 2013; Rai et al., 2014). This directly affect on economy as India is exporting fresh fruits and vegetables to different countries earning significant foreign exchange. About $16.27 \%$ of the foreign income comes from export of vegetables (Shivalingaswamy et al., 2006). Major pests attacking vegetables are, shoot and fruit borers causing damaging symptoms like withered terminal shoots, bore holes in stem and fill-up with fecal matter. They also cause shedding of flower buds, leaf drying, larvae and adult can bore the fruits and cause extensive damage. Leaf rollers which extensively feed on leaves, fold leaves from tip to downwards following withering and death of the leaves. Sap feeding insects include leaf hoppers, aphids suck the plant sap leading to yellowing, crinkling, downward curling and death of the foliage. Nymphs and adults are mostly involved in feeding (Shivalingaswamy et al., 2002). Another group of insects are root feeders include termite and grubs feed below ground tissues. They can tunnel upwards through stem and eat inner tissues. The affected plants completely wither and die soon. Some of the major insect pests of above category are presented in table 1 with extent of damage. India after signing of the general agreement of trade and tariff (GATT) of the world trade organization (WTO), more emphasis and importance was given to the use of ecofriendly pest management measures. Biological control is being integrated with other control measures because different methods are effective at different times, location under the influence of environmental conditions.

\section{Need for biological pest control in India}

The production and productivity of food grains reached 283 million tons during 201819. Along with food grains, the vegetable production also reached a record high of 187474 ('000MT) (indiaagristat.com). This is very much essential to meet the growing population of the country and their food demands. Beyond good production and productivity of agriculture and horticulture produce, the farmers often facing number of problems including high application of inputs especially chemical fertilizers and nutrients to get good yield and control of various insect pest and diseases. This has lead to the high cost of cultivation and investment which will reflect yield and monitory returns. On the other hand, the chemical pesticides and fertilizers have created environmental pollution and also affect human and animal life. This has led to considerable changes in attitude of farmers towards use of pesticides and switching over to alternate and ecofriedly approach. One such option is biological control where number of agents integrated into IPM practice for successful management of pests. Here no microorganism or beneficial insects will deliberately introduced or 
manipulated for biological control. The potential agents will be tested repeatedly under controlled conditions against a target pest followed by mass production and release for commercial purpose (Hodek et al., 2012). Advantages of biological pest control are given in Fig.1.

\section{Management using biological control agents}

Most of the plant protection measures in India are depends exclusively on chemical pesticides. The farmers are using pesticides making it a calendar based application. Since, the vegetable crops are high value crops; a small infestation can cause quality loss and reduction in monitory returns. Hence, the farmers repeatedly spraying insecticides even though the pest level is bellow ETL. This has become a common practice over the years by vegetable growers. Unknowingly they are destroying natural flora and fauna along with killing beneficial insects like predators, parasitoids and bees. Therefore it is absolutely necessary for the farmers to use biological control agents to conserve these beneficial insects along with safeguarding environment (Altieri et al., 2005; Mahr et al., 2008; Halder et al., 2011). During past few decades, a steady progress has been made in India towards biological control of insect pests. But, this needs to be aggravated in terms of searching more and more natural enemies, and microbial bio control agents for efficient management of insect pests in vegetable crops starting from laboratory tests, mass production and release for a specific pest. Detailed list of biological control agents used in vegetable pest control are given in Table 2 .

\section{Predators}

Predator insects are the beneficial insects which directly kill and feed on pests. Common predatory insects include lacewings, ladybird beetles, carabid beetles, staphylinid (rover) beetle, syrphid (hover) flies, minute pirate bugs, nabid bugs, big-eyed bugs, spiders and preying mantids. Ladybird beetles are recognized for their predatory behavior on many pests for centuries in United States (http://edis.ifas.ufl.edu). Adult and larvae of ladybird beetle feed on a number of small, soft-bodied insects, their eggs and larvae. Most of the predators are not host specific. They can feed on a number of pests including plant pests and insects eating on organic matter also. Predators are generally have chewing and sucking type of mouth parts and some types they have have both the types (Sampaio et al., 2010). Some of the insect orders have exclusively predatory insects. Ex: The order Odonata has dragon flies, where aquatic nymphs are predatory, and breath through gills. Whereas adults are excellent fliers captures their prey during flight from crop fields. Another order is Mantodea which have praying mantids. They are the excellent hunters of their prey by hiding on leaves and plant surface to confuse their prey. They have strong modified front legs to capture their prey. Similarly order Neuroptera have lacewings and ant-lions where, all the larvae are predators and adults feed on pollen and nectar (Sampaio et al., 2010). Order Diptera have rover flies which have similar mechanism of dragon flies to catch their prey. Other orders is Coleoptera (Coccinellids) having lady bird beetles which are the excellent predators. Many of the mite species belong to phytoseidae also reported to have predatory action. They are the important natural enemies of other mites.

\section{Parasitoids}

Parasitoids are the organisms which live and feed inside or on the host. The parasites can develop inside or outside of an insect's body. Only immature stage of the parasites feed on insect host. Adult females of certain parasites 
(such as many wasps that attack scale and whiteflies) feed on and their hosts providing an easily available source of biological control (Sanda and Sunusi, 2014). Based on the stage of prey that a parasite attacks, they are categorized into egg parasitoids which have whole development within the egg of other insect. Egg-larval parasitoid is the one that has oviposition within egg of the host, but its development completed in the insects larvae. Other parasitoids are larval, pupal and larvae-pupae. In some cases, adult stage of the insects also used as host by the parasitoid Sampaio et al., (2010). When the parasitoid develops on the host, it is called ectoparasitoid and when it develops inside it is called endoparasitoid. Most of the parasitic insects belong to order Diptera (flies) or Hymenoptera (Wasps). Parasitic wasps occur in three dozen Hymenoptera families. Ex: Aphidiinae (subfamily of Braconidae) attack aphids that are pests in most of the crops.

Table.1 Major insect pests attacking vegetable crops and their extent of damage in India

\begin{tabular}{|c|c|c|c|c|}
\hline $\begin{array}{l}\text { Sl } \\
\text { No }\end{array}$ & Pest & Crop infested & Parts damaged & $\begin{array}{l}\text { Extent of } \\
\text { damage }\end{array}$ \\
\hline 1 & Fruit borer (Helicoverpa armigera) & Tomato & Fruits & $24-73 \%$ \\
\hline 2 & Fruit and shoot borer (L. arbonalis) & Brinjal & Shoot and fruits & $11-93 \%$ \\
\hline \multirow[t]{2}{*}{3} & Thrips ( S. dorsalis) & \multirow[t]{2}{*}{ Chilli } & Leaves and fruits & $12-90 \%$ \\
\hline & $\begin{array}{l}\text { Mites (Polyphagotarsonemus latus } \\
\text { (Banks)) }\end{array}$ & & Leaves and fruits & $34 \%$ \\
\hline \multirow[t]{4}{*}{4.} & Fruit borer (Helicoverpa armigera) & \multirow[t]{4}{*}{ Bhendi/Okra } & Fruits & $22 \%$ \\
\hline & Leafhopper (A. bigutella bigutella) & & Leaves & $54-66 \%$ \\
\hline & Whitefly (B. tabaci) & & Leaves & $54 \%$ \\
\hline & Shoot and fruit borer (E. vittella) & & Shoot and fruits & $23-54 \%$ \\
\hline \multirow[t]{5}{*}{5.} & Diamond back moth $(P$ xylostella $)$ & \multirow[t]{5}{*}{ Cabbage } & Head & $17-99 \%$ \\
\hline & Cabbage caterpillar (P brassicae $)$ & & Leaf and head & $69 \%$ \\
\hline & $\begin{array}{l}\text { Cabbage leafwebber (Crocidolomia } \\
\text { binotalis) }\end{array}$ & & Leaves & $28-51 \%$ \\
\hline & Cabbage borer (H. undalis) & & Head & $30-58 \%$ \\
\hline & $\begin{array}{l}\text { Cabbage Butterfly } \quad \text { (Pieris } \\
\text { rapae/brassicae) }\end{array}$ & & Head & $40-68.5 \%$ \\
\hline 6. & Fruit fly (B. cucurbitae) & $\begin{array}{l}\text { Cucurbits } \\
\text { (Cucumber, Ivy } \\
\text { gourd, Musk melon, } \\
\text { Snake gourd, } \\
\text { Sponge gourd) }\end{array}$ & Fruits & $20-100 \%$ \\
\hline \multirow[t]{4}{*}{7} & Aphid (Myzus persicae (Sulzer)) & \multirow[t]{4}{*}{ Potato } & Leaves & $3-6 \%$ \\
\hline & Tobacco caterpillar $(S$. litura $)$ & & Leaves & $4-8 \%$ \\
\hline & $\begin{array}{l}\text { Potato tubermoth (Phthorimaea } \\
\text { operculelella (Zeller)) }\end{array}$ & & Tubers & $6-9 \%$ \\
\hline & Mite (P.latus) & & Leaves & $4-27 \%$ \\
\hline
\end{tabular}

Modified from, Shivalingaswamy et al., (2002); Dhillon et al., (2005); Satpathy et al., (2005); Raju et al., (2007); Singh et al., (2007); Ghosal et al., (2012); Rai et al., (2014), Sharma et al., (2017) 
Table.2 List of biological control agents used for management of insect pests of vegetable crops

\begin{tabular}{|c|c|c|c|c|c|}
\hline \multirow{3}{*}{$\begin{array}{l}\text { Sl } \\
\text { No }\end{array}$} & \multirow{3}{*}{$\begin{array}{l}\text { Name of } \\
\text { the } \\
\text { Vegetable }\end{array}$} & \multirow[t]{3}{*}{ Insect Pest } & \multicolumn{3}{|c|}{ Biological control agents } \\
\hline & & & Predators & \multirow[t]{2}{*}{ Parasitoids } & \multirow[t]{2}{*}{ Microbial agetns } \\
\hline & & & $\begin{array}{l}\text { Predatory bird species are: King } \\
\text { crow, common minah, }\end{array}$ & & \\
\hline & & & $\begin{array}{l}\text { Common predatory insects: Wasps, } \\
\text { dragon fly, spiders, robber fly, reduviid } \\
\text { bug, praying mantis, fire ants. }\end{array}$ & & \\
\hline \multirow[t]{2}{*}{1} & \multirow[t]{2}{*}{ Bhendi } & $\begin{array}{l}\text { Shoot and fruit } \\
\text { borer }\end{array}$ & $\begin{array}{l}\text { Chrysoperla carnea, Geocoris spp. } \\
\text { Eocanthecona furcellata. Coccinellid } \\
\text { beetles }\end{array}$ & $\begin{array}{l}\text { Trichogramma chilonis (egg), } \\
\text { Tetrastichus spp. (egg), Telenomus } \\
\text { spp. (egg), Chelonus blackburni } \\
\text { (egg-larval)Campoletis chlorideae } \\
\text { (larval), Goniophthalmus halli } \\
\text { (larval), Bracon spp. (larval) etc }\end{array}$ & $\begin{array}{l}\text { Ovomermis albicans, a } \\
\text { nematode }\end{array}$ \\
\hline & & White fly & $\begin{array}{l}\text { Mirid bug (Dicyphus hesperus), } \\
\text { (Geocoris sp) etc. }\end{array}$ & $\begin{array}{l}\text { Encarsia sp, Eretmocerus sp, } \\
\text { Chrysocharis pentheus }\end{array}$ & \\
\hline \multirow[t]{4}{*}{2} & \multirow[t]{4}{*}{ Tomato } & Aphids & $\begin{array}{l}\text { Aphidoletes aphidimyza (midge) and } \\
\text { Chrysoperla carnea (lacewing) }\end{array}$ & $\begin{array}{l}\text { Aphelinus abdominalis - Aphidius } \\
\text { colemani - Aphidius matricariae: } \\
\text { Aphidius ervi }\end{array}$ & $\begin{array}{l}\text { Verticillium lecanii } \\
\text { and Beauveria } \\
\text { bassiana (fungus) }\end{array}$ \\
\hline & & White fly & Macrolophus pygmaeus (bug), & $\begin{array}{l}\text { Eretmocerus mundus (specific to B. } \\
\text { tabaci), E. eremicus, E. Formosa. }\end{array}$ & $\begin{array}{l}\text { Verticillium leccani, } \\
\text { Paecilomyces } \\
\text { fumosoroseus, } \\
\text { Beauveria bassiana. }\end{array}$ \\
\hline & & $\begin{array}{l}\text { Leaf minor: } \\
\text { Tuta absoluta }\end{array}$ & $\begin{array}{l}\text { Nesidiocoris tenuis (bug), Macrolophus } \\
\text { pygmaeus (bug), Nabis pseudoferus }\end{array}$ & $\begin{array}{l}\text { Trichogramma pretiosum, } \\
\text { Trichogramma achaeae }\end{array}$ & $\begin{array}{l}\text { Bacillus thuringiensis } \\
\text { var. kurstaki, } \\
\text { Metarhizium and } \\
\text { anisopliae } \\
\text { Beauveria bassiana }\end{array}$ \\
\hline & & $\begin{array}{l}\text { Serpentine leaf } \\
\text { minor }\end{array}$ & $\begin{array}{l}\text { Lacewings, lady beetle, spiders, fire } \\
\text { ants, dragonfly, robber fly, praying }\end{array}$ & $\begin{array}{l}\text { Tetrastichus ovularum (egg), } \\
\text { Gronotoma micromorpha (larval and }\end{array}$ & \\
\hline
\end{tabular}




\begin{tabular}{|c|c|c|c|c|c|}
\hline & & & mantis etc. & pupal), Diglyphus sp. (larval), & \\
\hline \multirow[t]{2}{*}{3} & \multirow[t]{2}{*}{ Cabbage } & $\begin{array}{l}\text { Diamond } \\
\text { backmoth }\end{array}$ & Chrysoperla carnea, coccinellids, & $\begin{array}{l}\text { Trichogramma spp. (egg) Apanteles } \\
\text { glomeratus (larval), Bracon } \\
\text { gelechiae (larval), Bracon sp, } \\
\text { (larval), Mesochorus spp. (larval), } \\
\text { Brachymeria spp. (larval), Eriborus } \\
\text { spp. (larval), Diadegma } \\
\text { semiclausum, (larval) Cotesia } \\
\text { plutellae (larval), Diadromus } \\
\text { collaris (pupal), Diadromus spp. } \\
\text { (pupal), Brachymeria excarinata } \\
\text { (pupal)etc. }\end{array}$ & $\begin{array}{l}\text { Bacillus thuringiensis } \\
\text { (Bt). Paecylomyces } \\
\text { spp., Zoophthora } \\
\text { radican etc. }\end{array}$ \\
\hline & & Head borer & $\begin{array}{l}\text { Chrysoperla carnea, coccinellids, } \\
\text { (Geocoris sp), pentatomid bug } \\
\text { (Eocanthecona furcellata }\end{array}$ & $\begin{array}{l}\text { Trichogramma spp. (egg), Bracon } \\
\text { gelechiae (larval), B. Hebetor } \\
\text { (larval) etc. }\end{array}$ & $\begin{array}{l}\text { Paecylomyces } \text { spp., } \\
\text { Zoophthora radican } \\
\text { etc }\end{array}$ \\
\hline \multirow[t]{2}{*}{4.} & \multirow[t]{2}{*}{ Brinjal } & $\begin{array}{l}\text { Brinjal shoot } \\
\text { and fruit borer }\end{array}$ & $\begin{array}{l}\text { Mirid bug (Campyloneura sp), lady } \\
\text { bird beetles (Cheilomenes sexmaculata, } \\
\text { Coccinella septempunctata-seven } \\
\text { spotted, Brumoides suturalis-three } \\
\text { striped), lacewing (Chrysoperla } \\
\text { carnea), }\end{array}$ & $\begin{array}{l}\text { Trichogramma chilonis (egg), } \\
\text { Pseudoperichaeta sp (larval), } \\
\text { Phanerotoma sp (larval), Itamoplex } \\
\text { sp (larval), } \\
\text { argenteopilosus (larval), Diadegma } \\
\text { apostata, Pristomerous testaceus, } \\
\text { Trathala flavo-orbitalis (larval and } \\
\text { pupal), Cremastus sp (larval), } \\
\text { Bracon greeni (larval), Iphiaulax sp } \\
\text { (larval), Goryphus nursei (pupal) } \\
\text { etc. }\end{array}$ & \\
\hline & & Aphids & $\begin{array}{l}\text { Anthocorid bugs/pirate bugs (Orius } \\
\text { spp.), mirid bugs, syrphid/hover flies, } \\
\text { green lacewings (Mallada basalis and } \\
\text { Chrysoperla carnea), predatory } \\
\text { coccinellids (Stethorus punctillum), } \\
\text { staphylinid beetle (Oligota spp.), }\end{array}$ & $\begin{array}{l}\text { Aphidius colemani (adults and } \\
\text { nymphs), Diaeretiella spp. (adults } \\
\text { and nymphs), Aphelinus spp. (adults } \\
\text { and nymphs) etc. }\end{array}$ & \\
\hline
\end{tabular}




\begin{tabular}{|c|c|c|c|c|c|}
\hline & & & $\begin{array}{l}\text { predatory cecidomyiid fly (Aphidoletis } \\
\text { aphidimyza) and predatory gall midge, } \\
\text { (Feltiella minuta), }\end{array}$ & & \\
\hline & & Mites & $\begin{array}{l}\text { Anthocorid bugs (Orius spp.), mirid } \\
\text { bugs, syrphid/hover flies, green } \\
\text { lacewings (Mallada basalis and } \\
\text { Chrysoperla carnea), predatory mites } \\
\text { (Amblyseius alstoniae, A. womersleyi, } \\
\text { A. fallacies and Phytoseiulus } \\
\text { persimilis), predatory coccinellids } \\
\text { (Stethorus punctillum), staphylinid } \\
\text { beetle (Oligota spp.), predatory } \\
\text { cecidomyiid fly (Anthrocnodax } \\
\text { occidentalis), predatory gall midge } \\
\text { (Feltiella minuta) etc }\end{array}$ & & \\
\hline & & Leaf hopper & $\begin{array}{l}\text { Coccinellids, Distina albino, } \\
\text { Chrysoperla spp., mired bug (Dicyphus } \\
\text { hesperus), big-eyed bug, (Geocoris } \mathrm{sp}) \\
\text { etc. }\end{array}$ & $\begin{array}{l}\text { Lymaenon empoascae (egg), } \\
\text { Anagrus flaveolus, Stethynium } \\
\text { triclavatum }\end{array}$ & \\
\hline \multirow[t]{4}{*}{6} & \multirow[t]{4}{*}{ Potato } & Tuber moth & $\begin{array}{l}\text { Lacewing, red ant, ladybird beetle, } \\
\text { spider, robber fly, dragonfly etc. }\end{array}$ & $\begin{array}{lrr}\text { Chelonus } & \text { blackburni, } & \text { Copidosoma } \\
\text { koehleri, } & \text { Trichogrammar spp., } \\
\text { Apanteles } & \text { sp., } & \text { Pristomerus } \\
\text { vulnerator etc. } & \end{array}$ & \\
\hline & & Leaf minor & $\begin{array}{l}\text { Lacewing, ladybird beetle, spider, red } \\
\text { ant etc }\end{array}$ & $\begin{array}{l}\text { Chrysocharis pentheus, Gronotoma } \\
\text { micromorpha, Diglyphus isaea etc }\end{array}$ & \\
\hline & & White fly & $\begin{array}{l}\text { Ladybird beetle, lacewing, spider, } \\
\text { hover fly, reduviid bug, robber fly etc. }\end{array}$ & $\begin{array}{l}\text { Encarsia formosa, Eretmocerus spp. } \\
\text { etc. }\end{array}$ & \\
\hline & & Aphids & $\begin{array}{l}\text { Ladybird beetle, lacewing, spider, } \\
\text { hover fly et }\end{array}$ & $\begin{array}{l}\text { Lysiphlebus sp, Diaeretiella sp., } \\
\text { Aphelinus sp., Aphidius colemani } \\
\text { etc. }\end{array}$ & \\
\hline \multirow[t]{2}{*}{7} & \multirow[t]{2}{*}{ Cucurbits } & $\begin{array}{l}\text { Cucurbit fruit } \\
\text { fly }\end{array}$ & & Opius fletcheri (pupal) etc & \\
\hline & & Pumpkin beetle & Pennsylvania leather & Celatoria setosa (grub) etc. & Steinernema riobravis \\
\hline
\end{tabular}




\begin{tabular}{|c|c|c|c|c|c|}
\hline & & & (Chauliognathus pensylvanicus) & & \\
\hline & & $\begin{array}{l}\text { Serpentine leaf } \\
\text { miner }\end{array}$ & $\begin{array}{l}\text { Lacewings, lady beetle, spiders, fire } \\
\text { ants, dragonfly, robber fly, praying } \\
\text { mantis etc. }\end{array}$ & $\begin{array}{l}\text { Gronotoma micromorpha (larva and } \\
\text { pupa), Diglyphus sp. (larva), Opius } \\
\text { sp. (pupal) Chrysocharis sp., } \\
\text { Neochrysocharis formosa (Larval) } \\
\text { etc }\end{array}$ & \\
\hline & & Aphids & $\begin{array}{l}\text { Anthocorid bugs/pirate bugs (Orius } \\
\text { spp.), mirid bugs, syrphid/hover flies, } \\
\text { green lacewings (Mallada basalis and } \\
\text { Chrysoperla carnea), predatory } \\
\text { coccinellids (Stethorus punctillum), } \\
\text { staphylinid beetle (Oligota spp.), } \\
\text { predatory cecidomyiid fly (Aphidoletis } \\
\text { aphidimyza) and predatory gall midge, } \\
\text { (Feltiella minuta), }\end{array}$ & $\begin{array}{l}\text { Aphidius colemani, Diaeretiella spp. } \\
\text { Aphelinus spp. etc }\end{array}$ & \\
\hline 8 & Chillies & Aphids & $\begin{array}{l}\text { Anthocorid bugs/pirate bugs (Orius } \\
\text { spp.), mirid bugs, syrphid/hover flies, } \\
\text { green lacewings (Mallada basalis and } \\
\text { Chrysoperla carnea), predatory } \\
\text { coccinellids (Stethorus punctillum), } \\
\text { staphylinid beetle (Oligota spp.), } \\
\text { predatory cecidomyiid fly (Aphidoletis } \\
\text { aphidimyza) and predatory gall midge, } \\
\text { (Feltiella minuta), }\end{array}$ & $\begin{array}{l}\text { Aphidius colemani, Diaeretiella } \\
\text { spp., Aphelinus spp. etc. }\end{array}$ & \\
\hline & & $\begin{array}{l}\text { Spider mites } \\
\text { and yellow } \\
\text { mites }\end{array}$ & $\begin{array}{l}\text { Anthocorid bugs (Orius spp.), } \\
\text { Amblyseius ovalis, mirid bugs, } \\
\text { syrphid/hover flies, green lacewings } \\
\text { (Mallada basalis and Chrysoperla } \\
\text { carnea), predatory mites (Amblyseius } \\
\text { alstoniae, A. womersleyi, A. fallacies } \\
\text { and Phytoseiulus persimilis), predatory } \\
\text { coccinellids (Stethorus punctillum), } \\
\text { staphylinid beetle (Oligota spp.), }\end{array}$ & & $\begin{array}{l}\text { Beauveria bassiana } \\
\text { (entomo pathogen) }\end{array}$ \\
\hline
\end{tabular}




\begin{tabular}{|c|c|c|c|c|c|}
\hline & & & $\begin{array}{l}\text { predatory cecidomyiid fly } \\
\text { (Anthrocnodax occidentalis) } \\
\text { predatory gall midge (Feltiella minuta) } \\
\text { etc. }\end{array}$ & & \\
\hline & & Thrips & $\begin{array}{l}\text { Scolothrips indicus and } \\
\text { Franklinothrips megalops }\end{array}$ & & \\
\hline & & Gall midge & & $\begin{array}{lll}\text { Eurytoma } & \text { spp. } & \text { (larval-pupal } \\
\text { parasitoid), Bracon spp. ( larval } \\
\text { parasitoid), Dinarmus spp (larval } \\
\text { parasitoid) }\end{array}$ & \\
\hline & & Hadda beetle & & $\begin{array}{lll}\begin{array}{l}\text { Pediobius } \\
\text { endoparasitoid) }\end{array} & \text { fovelatus } & \text { (larval }\end{array}$ & Metarhizium anisopliae \\
\hline 9 & Onion & Thrips & $\begin{array}{l}\text { Aelothrips spp. Coccinellids } \\
\text { Galendromus occidentalis, lacewings } \\
\text { (Chrysopa spp., Chrysoperla spp.) } \\
\text { Amblyseius cucumeris and Amblyseius } \\
\text { barkeri. A. cucumeris (mites) }\end{array}$ & $\begin{array}{l}\text { Ceranisus menes, Orius spp. and } \\
\text { Anthocoris spp }\end{array}$ & $\begin{array}{l}\text { Beauveria bassiana } \\
\text { Metarhizium } \\
\text { anisopliae (Fungus) } \\
\text { Heterorhabditis indica } \\
\text { Steinernema } \\
\text { carpocapsae; } \\
\text { Heterorhabditis } \\
\text { bacteriophora (EPN) }\end{array}$ \\
\hline & & Maggot & $\begin{array}{l}\text { Braconid wasp Aphaereta pallipes, } \\
\text { Staphylinid and Aleochara bilineata }\end{array}$ & & \\
\hline
\end{tabular}

Source: Bora and Langthasa (1995); Basavraj et al., 2010; Vishwakarma et al., (2011); James et al., 2007; Garcia-del-Pino et al., 2013; Azazy et al., 2018; Mishra et al., 2014; Gandhi et el., 2016; Mahr et al., 1993;Nath et al., 2020;Pathak and Singh, 1997; 


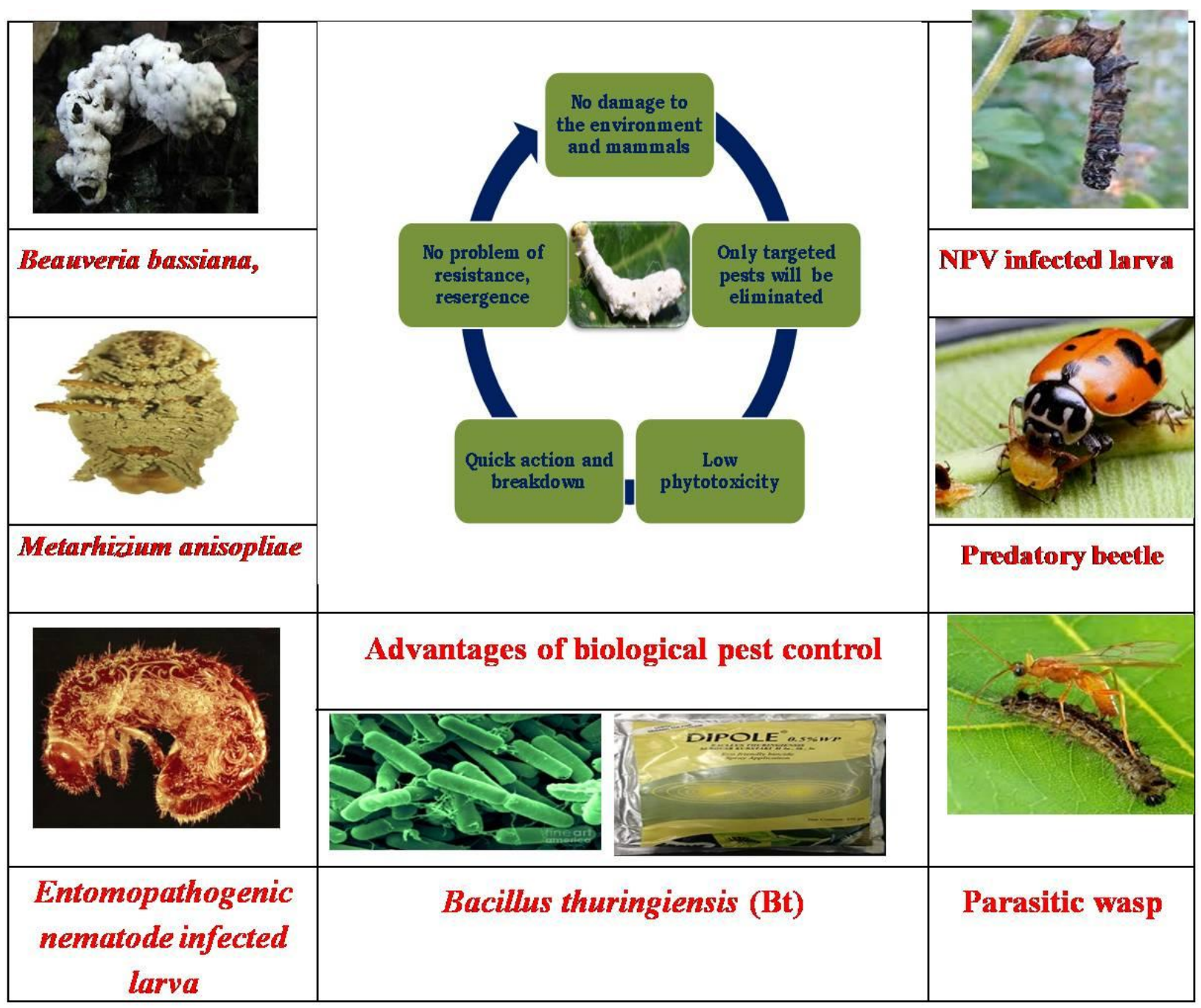

Other family is Trichogrammatidae, here pasitization is observed on eggs. Aphilinidae, Encyrtidae, Eulophidae and Ichneumonidae are the other families' parasites on insect pests (Flint and Dreistadt, 1998). There are 37 species of parasitoids known on Helicoverpa armigera India and only 8 species are important. $80 \%$ of the egg parasisitism was recorded by Trichogramma spp. on $H$. armigera infesting tomato (Manjunath 1989). The adult female wasp lays egg in each aphid host; the eggs develop into larva and feed on aphis and kills aphid. The parasitized aphid will die and turn into mummies. Caterpillar parasites include the Hyposoter exiguae wasp lays eggs on armyworm eats the insect host and kills it. Tachinid flies parasitize a number of insects. They lay eggs on caterpillar that will hatch and bore into the host (Hein et al., 2004; Dresistadt et al., 2004).

\section{Microbial biocontrol agents}

Just like plant pathogens, these are microbial agents belongs to fungi, bacteria, protozoa, virus, actinomycetes and nematodes which attack insect pests and kill them. Innudative application can be followed by formulating insect-pathogenic fungi (Metarhizium, Beauveria, Paecilomyces), insect-pathogenic bacteria (Bacillus thuringiensis-Bt), entamopathogenic nematodes (Heterorhabditis and Steinernema) and viruses (nuclear polyhedrosis virus-NPV and 
granulosis viruses (GV) (Flint and Dreistadt 1998). The fungal biocontrol agents belong to 12 classes within six phyla of the major groups like Laboulbeniales, Pyrenomycetes, Hyphomycetes and Zygomycetes. Many of the promising biocontrol agents have been ommercialized globally. They have been proven their efficacy on insect species belonging to Lepidoptera, Homoptera, Coleoptera, Orthoptera and Mites. Majority of the bacterial biological control agents are Bacillus thuringiensis based $\mathrm{Bt}$ formulations. In cabbage they are being used in two formulations like Bt kurstaki and Bt aizawai as control of diamond back both (DBM) and other defoliating lepidopteran insects (Shelton et al., 2007). These formulations are highly specific and very effective against target pests without any impact on natural enemies. Most of the formulations are spore-crystal mixtures having toxins (Btk-Cry1Aa, Cry 1Ab, Cry 1Ac. Cry 2a2A and Cry 2B; Bta;Cry 1Aa, Cry $1 \mathrm{Ab}$, CryIC, Cry ID and Cry 2B toxins) (Heckel et al., 2004; Grzywacz et al., 2010). Among the fungal biocontrol agenst, Acomycetes species like Beauveria bassiana, Metarhizium anisopliae sensulato, Nomuraea rileyi, Lecanicillium spp., gained much more attention during the past 30-50 years. There are more than 300 commercial products available in world market (Faria and Write 2007)

\section{Formulation of biocontrol agents as biopesticides}

There is an urgent need for reducing the usage of chemical pesticides in agriculture and promote sustainable biocontrol strategy. The usage of biological control agents or insect biopesticides has aroused increasing interest because of their ecological advantages (Roger 2012). However their commercialization lies very backward as we do not have proper regulatory framework for their registration, commercialization and utilization. The result is they occupy only marginal potion in plant protection products' market. There are three broad categories of bipesticides: microbial biopesticides, botanical pesticides and semiochemicals. The countries like Canada, USA, Eu, Australia and Brazil have shown interesting progress towards usage of biopesticides. India has shown slow growth, due to in part adequate legislation, a lack of capacity, and the week implementation of policies related to biopesticides and biological control agents (Arjjumend and Koutouki 2018). This leads, manufacturers and importers of biopesticides and biocontrol agents face multiple legal frameworks and procedural challenges (Arjjumend and Koutouki 2018). A shift in the legal framework from a focus on chemical pesticides to biological agents would also complement the country's environmental and sustainability goals. A number of organizations including, ICAR institutions and state agricultural universities are working on biological control of insect pests through a outstretch program. Major centers are, ICARIIPR, Kanpur and BHU (Uttar Pradesh), GBPUAT, Pantnagar (Uttarakhand), ICARIISR, Calicut (Kerala), RRL, SAKUAST, Srinagar (Jammu and Kashmir), TNAU, Coimbatore (Tamil Nadu), AAU, Jorhat (Assam), AAU, Anand (Gujarat), UAS Dharwad (Karnataka), RARS and ICARCTRI, Rajahmundry (Andhra Pradesh), PDKV, Akola (Maharashtra), OUAT, Bhubaneswar (Odisha), ICAR-CARI (Andaman and Nicobar), NIPHM Hyderabad (Telangana) and other institutes. These institutes have come up with many biocontrol technologies which are useful to the farmers.

In conclusion, vegetables being high value and low volume crops suffer from a number of insect pests. They not only reduced yield but also deteriorates quality. Vegetables are used in a number of ways either consumed fresh as salads or after cooking. The pesticide 
consumption of vegetable crops is higher than any other crops. The ill-effects of pesticides on environment and human health switched the interest towards biological control. Biological control is generally regarded as most effective and sustainable way of pest management in vegetable crops. Conservation of natural enemies, predators, parasitoids and microbial biocontrol agents can sustain the pest management alternative to chemical pesticides. Though biological control will not control all the insects at a time, it should be an integrative component of integrated pest management.

\section{Abbreviations}

EIL: Economic Injury Level.

APEDA: Agricultural and Processed Food Products Export Development Authority

NHB: National Horticulture Board

IPM: Integrated Pest Management

GATT: General Agreement on Trade and Tariff

WTO: World Trade organization

ICAR: Indian Council of Agricultural Research

IIPR: Indian Institute of Pulses Research

BHU: Banaras Hindu University

SKUAST: Sher-e-Kashmir University of Agricultural Sciences \& Technology

TNAU: Tamil Nadu Agricultural University

UAS: University of Agricultural Sciences

RARS: Regional Agricultural Research Institute

CTRI: Central Tobacco Research Institute

AAU: Assam Agricultural University

AAU: Anand Agricultural University

IISR: Indian Institute of Spices research

CARI: Central Island Agricultural Research Institute

PDKV: Panjabrao Deshmukh Krishi Vidyapeet

OUAT: Orissa University of Agriculture and Technology

NIPHM: national Institute of Plant Health Management

\section{References}

Agricultural and Processed Food Products Export Development Authority (APEDA) (2020) Department of Commerce and Industry, Union Budget 2020-21, Press Information Bureau, Ministry of Statistics and Programme Implementation, Press Releases, Media Reports, Ministry of Agriculture and Farmers Welfare, Crisil.

Altieri M, CI Nichols, MA Fritz (2005) Manage insects on your farm: A guide to ecological strategies. Sustainable agriculture network handbook series book 7. (Available online at: http://www.sare.org/Learning-

Center/Books/Manage-Insects-on-YourFarm) (verified 25 April 2011).

Arjjumend H, Koutouki K (2018) Science of Biopesticides and Critical Analysis of Indian Legal Frameworks Regulating Biocontrol Agents. International Journal of Agriculture, Environment and Biotechnology Citation: IJAEB: 11(3): 563-571, June 2018

Basavaraj K, Sreenivas AG, Prabhuraj A et al., (2010) Seasonal incidence of chilli gall midge (Asphondylia capparis Rubsaaman.) and its parasitoids. Karnataka Journal of Agricultural Sciences 24 (4):555-557.

Borah RK, Langthasa S (1995) Incidence of thrips Scirtothrips dorsalis Hood in relation of date of transplanting on chilli in hill zone of Assam. PKV Res J 92:191-192

Dastagiri MB, Ramesh Chand TK, Immanuelraj CV et al., (2013) Indian Vegetables: Production Trends, Marketing Efficiency and Export Competitiveness. American Journal of Agriculture and Forestry 1-11. doi: 10.11648/j.ajaf.20130101.11.

Dhillon MK, Singh R, Naresh JS, Sharma HC (2005) The melon fruit fly, Bactrocera cucurbitae: A review of its biology and 
management. Journal of Insect Science 5:40.

Dreistadt SH, Flint ML, Clark JK (2004) Pests of Landscape Trees and Shrubs: An Integrated Pest Management Guide. 2nd ed. Oakland: Univ. Calif. Agric. Nat. Res. Publ. 3359.

Flint ML, Dreistadt SH (1998) Natural Enemies Handbook : The Illustrated Guide to Biological Pest Control. Oakland: Univ. Calif. Div. Agric. Nat. Res. Publ. 3386.

Gandhi K, Chinnasamy D, Sowrirajan R, Bangaru P, Subramaniam S (2016) IPM of tropical vegetable crops. pp 167-177.

Garcia-del-Pino F, Alabern X, Morton A (2013). Efficacy of soil treatments of entomopathogenic nematodes against the larvae, pupae and adults of Tuta absoluta and their interaction with the insecticides used against this insect. BioControl, 58(6):723-731. http://rd.springer.com/journal/10526.

Ghosal A, Chatterjee ML, Manna D (2012). Studies on some insecticides with novel mode of action for the management of tomato fruit borer (Helicoverpa armigera Hub.). Journal of Crop and Weed 8(2): 126-129.

Grzywacz D, Rossbach A, Rauf A etal (2010) Current control methods for diamondback moth and other brassica insect pestsand the prospects for improved management with lepidopteran-resistant $\mathrm{Bt}$ vegetable brassicas in Asia and Africa. Crop Protection 29: 68-79.

Halder J, Rai AB, Kodandaram MH, Dey D (2011) Pediobius foveolatus (Crowford):A promising bioagents against Epilachna vigitionctopunctata. Uttarakhand, 28 In. National Conference on Horti-business linking farmers with market. Held at Dehradun, 28-31May, 2011. Pp-133.

Heckel D, Tabashnik B, Liu YB et al., (2004)
Diamondback moth resistance to Bt. In: Endersby, N., Ridland, P.M. (Eds.),The Management of Diamondback Moth and Other Crucifer Pests pp. 61-66.

Hein KM, Van Driesche RG, Parella MP 2004. Bio Control in Protected Culture. (Ed). Ball Publishing, Batavia, Ill. 522 pp.

Hodek I, Van Emden HF, Honek A (2012) Ecology and behavior of ladybird beetles (Coccinellidae. WileyBlackwell, Chichester, UK.

James B, Godonou I, Atcha-Ahowe C etal (2007) Extending integrated pest management to indigenous vegetables. Acta. Hortic. 752, 89-93.

Mahr DL, Whitaker P, Ridgway NM (2008) Biological control of insects and mites: An introduction to beneficial natural enemies and their use in pest management. University of Wisconsin Cooperative Extension, No. A3842.

Mahr SE, Mahr DL, Wyman JA (1993) Biological control of insect pests of cabbage and other crucifers. North Central Regional Publication. 471.

Manjanuth TM, Bhatnagar VS, PawaR CS, Sitanatham S (1989). Economic importance of Heliothis spp. in India and an assessment of their natural enemies and host plants. In: King EG, Jackson RD (eds) Proceedings of the Workshop on Biological Control of Heliothis: Increasing the Effectiveness of Natural Enemies November 1985, New Delhi. New Delhi, India: Far Eastern Regional Research Office, US Department of Agriculture, 196-228.

Nath RK, Begum KH, Ahmed, P (2020) Integrated Pest Management of Okra (Abelmoschus esculentus L.). International Journal of Current Microbiology and Applied Sciences 9(01): $\quad$ 1725-1729.doi: https://doi.org/10.20546/ijcmas.2020.90 1.191 . 
National Horticulture Board (2018) National Horticulture Data base (http://nhb.gov.in).

Orr D (2009) Biological Control and Integrated Pest Management. In: Peshin R., Dhawan A.K. (eds) Integrated Pest Management: Innovation-Development Process. Springer, Dordrecht

Patnaik HP, Singh KM (1997) Efficacy of Bacillus thuringiensis Berliner and conventional insecticides against brinjal shoot and fruit borer under different spraying schedule. Orissa J. Hort. 25 (1): $18-21$

Pires LM, Marques EJ, Wanderley-Teixeira V, Teixeira ÂAC, Alves LC, Alves ESB(2009) Ultrastructure of Tuta absoluta parasitized eggs and the reproductive potential of females after parasitism by Metarhizium anisopliae. Micron 40(2):255-261.

Rai AB, Halder J, Kodandaram MH (2014) Emerging insect pest problems in vegetable crops and their management in India: An appraisal. Pest Management in Horticultural Ecosystems 20(2): 113-122.

Raju SVS, Bar UK, Uma Shankar, Kumar S (2007) Scenario of Infestation and Management of Eggplant Shoot and Fruit Borer, Leucinodes orbonalis Guen., in India. Resistant Pest Management Newsletter 16(2):14-16.

Roger CR (2012) Trends for Commercialization of Biocontrol Agent (Biopesticide) Products. In book: Plant Defence: Biological Control. DOI: 10.1007/978-94-007-1933-06

Sampaio MV, Bueno VHP, Silveira LCP et al., (2010) Tropical biology and conservation management - Vol. III Biological Control of Insect Pests In The Tropics .

Sanda NB, Sunusi M. (2014). Fundamentals of biological control of pests. International Journal of Chemical and
Biochemical Sciences. 1(6).

Satpathy S, Kumar A, Singh AK, Pandey PK (2005) Chlorfenapyr: A new molecule for diamondback moth (Plutella xylostella $\mathrm{L}$.) management in cabbage. Annals of Plant Protection Sciences 13: 88-90.

Sharma S, Kooner R, Arora R (2017) Insect Pests and Crop Losses. In: Arora R., Sandhu S. (eds) Breeding Insect Resistant Crops for Sustainable Agriculture. Springer, Singapore.

Shelton AM, Roush RT, Wang $\mathrm{P}$ et al., (2007) Resistance to insect pathogens and strategies to manage resistance: An update. In: Lacey L, Kaya HK(Eds.) Field Manual of Techniques in Invertebrate Pathology, second ed. Kluwer Academic Press pp. 793-811.

Shivalingaswamy TMS, Satpathy S, Banerjee MK (2002) Estimation of crop losses due to insect pests in vegetables, In: Resource Management in plant protection, Vol-I (Sarat Babu, B., Vara Prasad, K.S., Anita, K. Rao R.D., Chakraborty, S.K. and Chandukar, P.S. eds.) Plant Projection association of India, Hyderabad, pp: 24-31.

Shivalingaswamy TMS, Satpathy S, Rai AB, Rai M (2006) Insect pests of vegetable crops: Identification and management. Technical Bulletin No. 30, IIVR, Varanasi, pp- 15.

Singh SK, Singh AK, Singh HM (2007) Relative resistance of okra germplasm to shoot and fruit borer, Earias vittella Fabr. under field conditions. Journal of Applied Zoological Researches 18(2): 121-123.

Vanitha SM, Chaurasia SNS, Singh PM et al., (2013) Vegetable Statistics. Technical Bulletin No. 51, IIVR, Varanasi pp. 250.

Vishwakarma R, Prasad PH, Ghatak SS et al., (2011) Bio-efficacy of plant extracts and entomopathogenic fungi against 
Epilachna beetle, Henosepilachna vigintioctopunctata (Fabr icius) infesting bottle gourd. Journal of Insect Science 24(1): 65-70.

Krishnamoorthy A., Mani M. (2001) Biological Control of Pests of Vegetable Crops. In: Upadhyay R.K.,
Mukerji K.G., Chamola B.P. (eds) Biocontrol Potential and its Exploitation in Sustainable Agriculture. Springer, Boston, MA. https://doi.org/10.1007/978-1-46151377-3_21

\section{How to cite this article:}

Pati, P., S.K. Behera, S. Raghu and Annamalai, M. 2021. Biological Control of Insect Pests in Vegetable Crops: An Eco-friendly Approach. Int.J.Curr.Microbiol.App.Sci. 10(01): 1358-1373. doi: https://doi.org/10.20546/ijcmas.2021.1001.162 